\title{
Wahlprüfsteine 2021
}

\section{Deutscher Bibliotheksverband adressiert Wahlprüfsteine zur Bundestagswahl 2021 an politische Parteien}

https://doi.org/10.1515/bd-2021-0070

Im Vorfeld der Bundestagswahlen am 26. September 2021 hat der Deutsche Bibliotheksverband e.V. (dbv) Wahlprüfsteine mit seinen wichtigsten bibliothekspolitischen Anliegen an die politischen Parteien veröffentlicht. Anhand von sieben Themenschwerpunkten werden die im Deutschen Bundestag vertretenen Parteien gebeten, darzulegen, wie sie sich in der nächsten Legislaturperiode für diese Themen einsetzen werden.

Die Wahlprüfsteine des dbv behandeln folgende Themenbereiche:

1. Bibliotheken als öffentliche Orte der Begegnung und des Austauschs

2. Bibliotheken als Orte digitaler Bildung

3. Bibliotheken als Orte digitaler Teilhabe

4. Urheberrecht in Wissenschaft und Forschung

5. Umsetzung der Nachhaltigkeitsziele durch Einbindung von Bibliotheken

6. Erhalt des schriftlichen Kulturguts in Bibliotheken

7. Digitales Kulturerbe in Bibliotheken

Dazu Prof. Dr. Andreas Degkwitz, Bundesvorsitzender des dbv: „Bibliotheken vermitteln Zugang zu Informationen und Wissen sowie Lese-, Informations- und Medienkompetenz und ermöglichen damit Teilhabe an Bildung, Gesellschaft und Kultur. Um ihr Potenzial ausschöpfen und ihre Vorhaben umsetzen zu können, brauchen sie politische Unterstützung sowie gesicherte rechtliche und finanzielle Rahmenbedingungen. Die Wahlprüfsteine 2021 zeigen die Themen auf, die den Bibliotheken besonders auf den Nägeln brennen und für die sie auf Unterstützung aus der Politik angewiesen sind.“

Auch wenn Bibliotheken aufgrund des föderalen Prinzips in die unmittelbare politische Zuständigkeit der Länder und Kommunen fallen, wirkt die Bundespolitik durch ihre Gesetzgebungen auf die Arbeit der Bibliotheken ein und kann wichtige Impulse für eine moderne Bibliotheksentwicklung setzen.

Die Wahlprüfsteine können auf der Webseite des dbv heruntergeladen werden: https://www.bibliotheksverband.de/dbv/themen/wahlpruefsteine/ bundestagswahl.html.

Die Antworten der Parteien werden dort ab Juli veröffentlicht. 


\section{Der Deutsche Bibliotheksverband e.V. (dbv)}

Der Deutsche Bibliotheksverband e.V. (dbv) vertritt mit seinen mehr als 2.100 Mitgliedern bundesweit rund 10.000 Bibliotheken mit 25.000 Beschäftigten und 11 Mio. Nutzer^innen. Sein zentrales Anliegen ist es, Bibliotheken zu stärken, damit sie allen Bürger^innen freien Zugang zu Informationen ermöglichen. Der Verband setzt sich ein für die Entwicklung innovativer Bibliotheksleistungen für Wissenschaft und Gesellschaft. Als politische Interessensvertretung unterstützt der dbv die Bibliotheken insbesondere auf den Feldern Informationskompetenz und Medienbildung, Leseförderung und bei der Ermöglichung kultureller und gesellschaftlicher Teilhabe für alle Bürger`innen.

Mehr Informationen finden Sie unter www.bibliotheksverband.de.

\section{Pressekontakt}

Kristin Bäßler

Deutscher Bibliotheksverband

Leitung Kommunikation / Pressesprecherin

Tel.: + 49 (0)30 6449899 25 | E-Mail: baessler@bibliotheksverband.de 